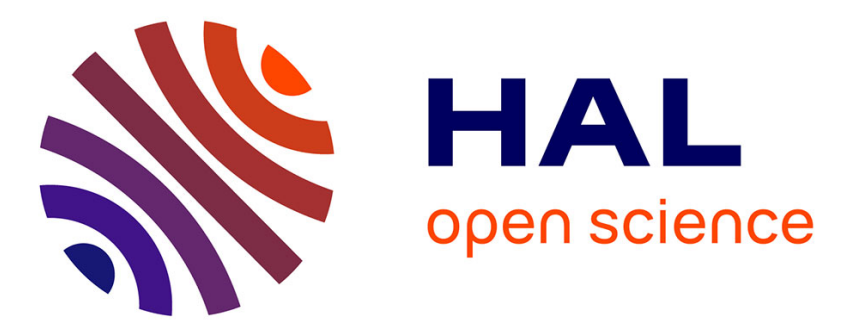

\title{
Propagating spatial and thematic uncertainties in mountain natural hazard assessment process
}

\author{
G. Dupouy, J.M. Tacnet, Franck Bourrier, F. Berger, N. Crimier, K. \\ Mekhnacha, M. Memier, E. Moulet-Vargas
}

\section{> To cite this version:}

G. Dupouy, J.M. Tacnet, Franck Bourrier, F. Berger, N. Crimier, et al.. Propagating spatial and thematic uncertainties in mountain natural hazard assessment process. Safety and Reliability of Complex Engineered Systems ESREL 2015, Sep 2015, Zurich, Switzerland. pp.261-268, 10.1201/9781315374987-9 . hal-02605957

\section{HAL Id: hal-02605957 https://hal.inrae.fr/hal-02605957}

Submitted on 16 Aug 2021

HAL is a multi-disciplinary open access archive for the deposit and dissemination of scientific research documents, whether they are published or not. The documents may come from teaching and research institutions in France or abroad, or from public or private research centers.
L'archive ouverte pluridisciplinaire HAL, est destinée au dépôt et à la diffusion de documents scientifiques de niveau recherche, publiés ou non, émanant des établissements d'enseignement et de recherche français ou étrangers, des laboratoires publics ou privés.

\section{(c)(1)}

Distributed under a Creative Commons Attribution| 4.0 International License 


\title{
Propagating spatial and thematic uncertainties in mountain natural hazard assessment process
}

\author{
G. Dupouy \& J.M. Tacnet \\ UR ETGR, Snow Avalanches Engineering and Torrent Control Research Unit, IRSTEA, \\ Grenoble-Alpes University, Saint Martin d'Heres, France \\ F. Bourrier \& F. Berger \\ UR EMGR, Mountain Ecosystems research unit, IRSTEA, Grenoble-Alpes University, Grenoble, France
}

\author{
N. Crimier \& K. Mekhnacha \\ ProBayes, Montbonnot, France
}

M. Memier \& E. Moulet-Vargas

Sintegra, Meylan, France

\begin{abstract}
Natural phenomena in mountains such as rockfalls cause severe damage to exposed population and assets. Numerical modelling is widely used to assess the hazard level (combination of phenomenon's intensity and frequency). This paper describes how innovative methods and tools are used to assess both thematic (related to the characteristics of the rockfalls) and spatial (related to the local topography) data uncertainties considering rockfall propagation. Those uncertainties are propagated in rockfall simulation models using classical Monte Carlo (probabilistic) and hybrid (possibilistic) approaches. Uncertainties related to the altimetric information, through Digital Elevation Model (DEM), are modelled by random fields using the new ModTer software, that can produce terrain simulations. This paper compares both uncertainty propagation approaches, taking into account spatialized uncertain variables, and proposes a sensitivity analysis describing the contribution of DEM variability on the global uncertainty.
\end{abstract}

\section{INTRODUCTION}

In mountains, natural phenomena such as rockfalls, put people and assets such as buildings, transport infrastructures) at risk. Rockfall can be defined as small mass movement that consists in the removal of a superficial boulder from a cliff face or a talus slope (Figure 1).

Risk is classically defined as a combination of hazard and vulnerability. Hazard relates to the intensity and frequency of phenomena. Direct vulnerability concerns physical damages and values assessment (of elements at risk) as a combination of spatial exposure and potential losses (Figure 2) (Tacnet 2009).

Risk reduction measures include mitigation actions which are based either on structural measures, such as civil engineering protection works (embankments, restraining nets etc.), or on non-structural measures, such as land-use control through risk zoning maps (Tacnet et al. 2014). Local authorities and infrastructure managers have often to take difficult decisions to choose between combinations of these structural and non-structural strategies according to their structural, functional and economic effectiveness (Carladous

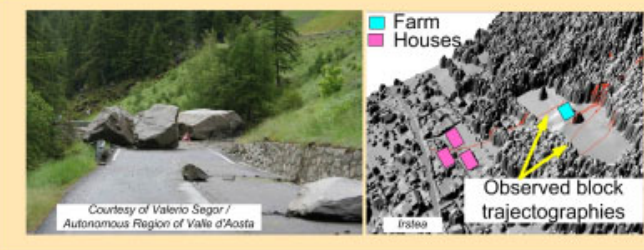

Figure 1. Rockfalls put people and infrastructures at risk.

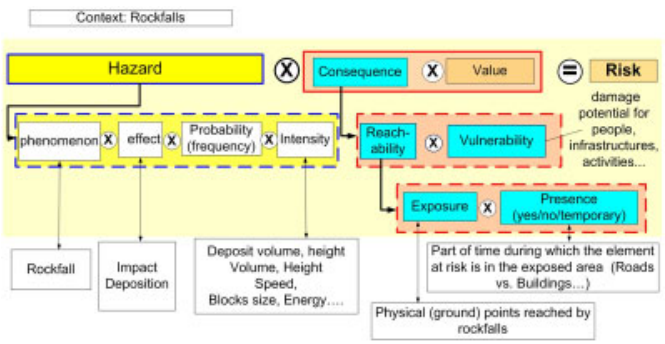

Figure 2. Risk assessment based on phenomenon thematic and spatial information. 


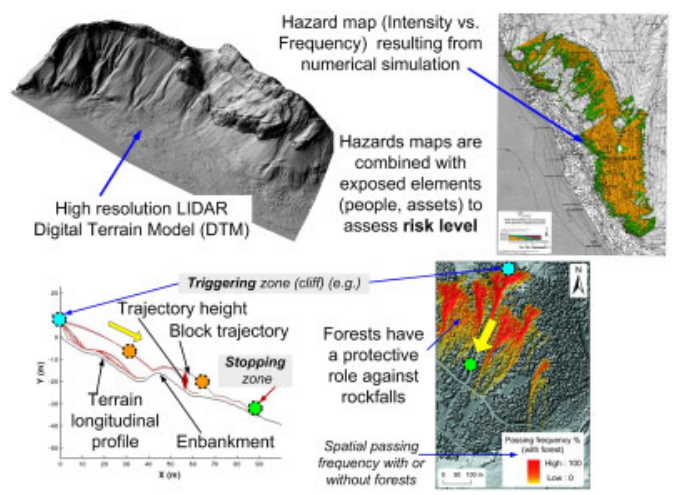

Figure 3. Spatial information is used to assess rockfall hazards and risks.

et al. 2015). However, risk mapping remains an essential objective for risk managers and numerical modelling is used to asses phenomena's extension and intensity. For rockfalls, trajectography modelling is used to represented the potential paths of blocks falling down from cliffs to the valleys (Figure 3 ).

In this context, expertise remains essential to help taking decisions. Each step of the expert assessment and decision processes depends on quality, availability and imperfection of information (such as imprecision, incompleteness, conflict or variability). Evaluating, propagating and tracing information quality according to sources' reliability is needed to take proper decisions and give an objective and realistic feedback about confidence level for expert assessments and resulting decisions. The risk analysis process uses quantitative and qualitative thematic information about natural environment (rockfall volume, geology, geomorphology, hydrology) but also spatial information related to topography. All this information come from several, more or less reliable, sources such as historical data, numerical models or expert assessments.

Numerical simulation results depend on the availability and the quality (or imperfection) of input data. Recent developments have introduced uncertainty analysis of those numerical simulations but have focused on thematic, scalar variables, such as block volume (Dupouy et al. 2015). In trajectography analysis, terrain topography has also an essential role and its uncertainty has to be considered in numerical modelling. The ModTer project aims first to assess spatial uncertainty related to available elevation data and, secondly, to analyse the influence of this uncertainty on global simulation results. A ModTer software has been developed (Crimier et al. 2016) to build DEM uncertainty models. A specific framework has been build to propagate both DEM uncertainty, modelled by the software, and thematic uncertainty (Figure 4).

This paper aims first to generalise the comparison of Monte Carlo and Hybrid uncertainty propagation results described in Dupouy et al. (2015), by including DEM variability as modelled by ModTer, and

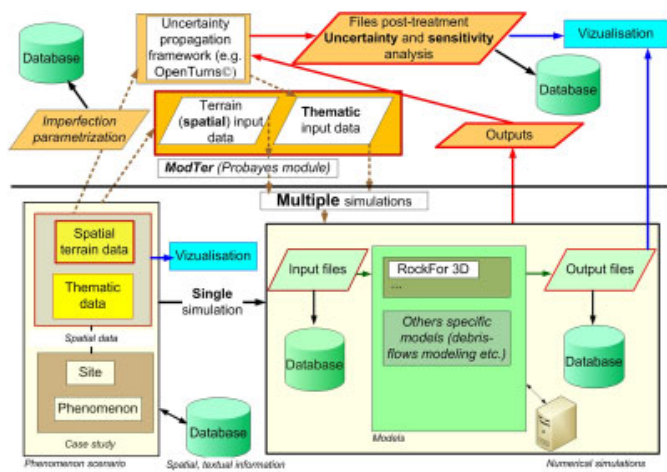

Figure 4. Spatial and thematic uncertainty propagation framework.

by focusing on spatialized variables of interest. It also provides a sensitivity analysis to evaluate the influence of DEM variability over the output uncertainty. The present first section introduces the context and needs. The second part recalls the main tools and method, and more specifically the possibilistic, so-called Hybrid propagation method, and the main outputs of the new ModTer software. It also details the description of the analysis. The third section presents the results of Monte Carlo and Hybrid propagation works, and sensitivity analysis. Finally the discussion analyses the results and concludes about perspectives.

\section{MATERIALS AND METHODS}

\subsection{Representations of imprecise information}

Let be a probability space $(\mathbb{R}, \mathcal{A}, P)$ and $X$ a random variable.

\subsubsection{Possibility theory}

The possibility theory (Zadeh 1978, Dubois et al. 2000) provides a tool to represent imprecise information. It models both variability and imprecision of $X$, by associating a possibility distribution $\pi$.

A possibility distribution is a mapping $\pi$ : $\mathbb{R} \rightarrow[0,1]$ such that $\sup _{x \in \mathbb{R}} \pi(x)=1$. It describes the potential likelihood of the values of $X$, and induces two measures, namely possibility $\Pi$ and necessity $N$ such that, for any $A \in \mathcal{A}$ :

$$
\begin{aligned}
& \Pi(A)=\sup _{x \in A} \pi(x) \\
& N(A)=\inf _{x \in \bar{A}} \pi(x)=1-\Pi(\bar{A})
\end{aligned}
$$

$\Pi(A)$ measures to which extent $A$ is plausible, $N(A)$ to which extent $\bar{A}$ is impossible. In its probabilistic interpretation, $\Pi(A)$ and $N(A)$ can be considered respectively as upper and lower bounds of the probability $P(A)$, defining a set of probability measures 
(Dubois and Prade 1992, de Cooman and Aeyels 1999):

$$
\begin{aligned}
& \mathcal{P}_{\pi} \quad=\{P \mid \forall A \in \mathcal{A}, \quad N(A) \leq P(A) \leq \Pi(A)\} \\
& =\{P \mid \forall A \in \mathcal{A}, \quad N(A) \leq P(A)\} \\
& =\{P \mid \forall A \in \mathcal{A}, \quad P(A) \leq \Pi(A)\}
\end{aligned}
$$

Given $\alpha \in[0,1]$, the $\alpha$-cut of $\pi$ is the set $E_{\alpha}=\{x, \pi(x) \geq \alpha\}$, and $P\left(E_{\alpha}\right) \geq 1-\alpha$ according to (2) and (4).

Thus, such a distribution, formally equivalent to fuzzy interval, reflects conveniently an expert assessment, expressed in terms of nested intervals $E_{1} \supset \cdots \supset E_{n}$, with decreasing confidence levels $\lambda_{1}>\cdots>\lambda_{n}$ (interpreted as $\left.P\left(E_{i}\right) \geq \lambda_{i}\right)$, one can deduce a possibility distribution $\pi$ whose the $\left(1-\lambda_{i}\right)$ cut is $E_{i}(1 \leq i \leq n)$.

Similarly to probability distributions, one can simulate a possibility distribution by sampling values $\alpha_{i}$ from an uniform law on $[0,1]$, getting the corresponding $\alpha_{i}$-cuts.

\subsubsection{Random sets}

A random set is defined by a mass function $m$ associated to a finite set $\mathcal{F}$ of measurable subsets of $\mathbb{R}$, called focal elements, such that $\sum_{E \in \mathcal{F}} m(E)=1$.

According to the theory of evidence introduced by Shafer (1976) and Smets and Kennes (1994), $m$ induces two measures, namely plausibility $P l$ and belief $B e l$ such that, for any $A \in \mathcal{A}$ :

$$
\begin{aligned}
& P l(A)=\sum_{E \cap A \neq \emptyset} m(E) \\
& \operatorname{Bel}(A)=\sum_{E \subseteq A} m(E)=1-\operatorname{Pl}(\bar{A})
\end{aligned}
$$

$P l(A)$ measures to which extent $A$ is plausible, $\operatorname{Bel}(A)$ to which extent $\bar{A}$ is impossible. In its probabilistic interpretation, $\operatorname{Pl}(A)$ and $\operatorname{Bel}(A)$ can be considered respectively as upper and lower bounds of the probability $P(A)$, defining a set of probability measures:

$$
\begin{aligned}
& \mathcal{P}_{m}=\{P \mid \forall A \in \mathcal{A}, \quad \operatorname{Bel}(A) \leq P(A) \leq P l(A)\} \\
& =\{P \mid \forall A \in \mathcal{A}, \quad \operatorname{Bel}(A) \leq P(A)\} \\
& =\{P \mid \forall A \in \mathcal{A}, \quad P(A) \leq P l(A)\}
\end{aligned}
$$

If focal elements are singletons, random sets encompass probability theory (in the discrete case). The case where focal elements are nested intervals corresponds to the possibility theory context.

\subsubsection{Probability boxes}

Considering a couple of cumulative distribution functions $(\underline{F}, \bar{F})$ with $\underline{F} \leq \bar{F}$, the related probability box (P-box) is defined as the set of probability measures :

$$
\mathcal{P}_{\underline{F} \leq \bar{F}}=\{P \mid \forall x \in \mathbb{R}, \quad \underline{F}(x) \leq F(x) \leq \bar{F}(x)\}
$$

The gap between $\underline{F}$ and $\bar{F}$ reflects the imprecision of the available information.

Thus, given a probability set deduced from a mass function $\mathcal{P}_{m}$, one can consider the P-box such that $\underline{F}(\cdot)=\operatorname{Bel}((-\infty, \cdot])$ and $\bar{F}(\cdot)=\operatorname{Pl}((-\infty, \cdot])$. Though this P-box is more intuitive to represent imprecise probability, it is obviously a looser approximation of the initial probability family (we only consider very specific events). Yet it remains adapted to bound quantiles or failure probabilities, which are common criteria of interest in risk analysis.

\subsection{Hybrid uncertainty propagation}

Given a mathematical model $G: \mathbb{R}^{n} \rightarrow \mathbb{R}^{p}$ and $Y=G\left(X_{1}, \ldots, X_{n}\right)$ the variable of interest. Let us suppose that we want to evaluate a high-order quantile (e.g. 0.975-quantile), from a perspective of structure dimensioning.

The Hybrid uncertainty propagation method (Baudrit et al. 2006) mixes probabilistic convolution with fuzzy calculus. It leans on probability, possibility (Zadeh 1978, Dubois et al. 2000) and random sets theories (Dempster 1967, Shafer 1976), used as practical tools for coding imprecise probabilities (Dubois et al. 2000). It combines usual Monte-Carlo method and interval analysis by $\alpha$-cuts.

\subsubsection{Sampling}

Let us suppose that input variables $X_{1}, \ldots, X_{k}(k \leq n)$ are precise enough to be reasonably modelled by an unique joint probability distribution $p_{1: k}$.

$X_{k+1}, \ldots, X_{n}$ are imprecisely known (few or imprecise measured data, expert assessments etc.), so that associating them an unique probability distribution implies to make strong assumptions. Thus, they are respectively associated to possibility distributions $\pi_{k+1}, \ldots, \pi_{n}$.

A Hybrid realization is designed by the association of:

- a random tuple $x_{1: k}$, stemming from $p_{1: k}$;

- a $(n-k)$-hypercube $E_{k+1: n}$, stemming from the $\alpha$-cuts $(\alpha \in[0,1]$ being a random degree of confidence) of the possibilistic variables such that $E_{k+1: n}=E_{k+1}^{\alpha} \times \cdots \times E_{n}^{\alpha}$.

Then, a Hybrid $M$-sample is designed by $M$ such random realizations.

\subsubsection{Optimization and propagation}

For each realization $\left(x_{1: k}^{i}, E_{k+1: n}^{i}\right), 1 \leq i \leq M$, one optimizes $G$ on $E_{k+1: n}^{i}$ to get an output interval $\left[y_{\text {inf }}^{i}, y_{\text {sup }}^{i}\right]$. A mass $\frac{1}{M}$ is assigned to this interval, resulting a random set on $Y$.

Due to the considerable computational cost of such optimization works, an usual hypothesis is to assess a monotone relationship between as much imprecise inputs as possible, and the variable of interest. Hence, the dimension of optimization space is reduced. 


\subsubsection{Post-treatment}

At this point, according to random sets theory, the random set on $Y$ can be interpreted in terms of imprecise probability. The probability of any event $A$ on $Y$ can be bound by the dual measures $\operatorname{Bel}(A)=\sum_{E \subseteq A} m(E)$ and $P l(A)=\sum_{E \cap A \neq \emptyset} m(E)$.

One can consider for convenience the related $\mathrm{P}$ box, such that $\underline{F}=\operatorname{Bel}((-\infty, \cdot])$ and $\bar{F}=\operatorname{Pl}((-\infty, \cdot])$. It is proved that $\underline{F}$ and $\bar{F}$ coincide with the empirical cumulative distribution functions of $\left(y_{\text {sup }}^{i}\right)_{i}$ and $\left(y_{\text {inf }}^{i}\right)_{i}$ respectively (ref).

More specifically, one can bound any $\gamma$-quantile by the estimated $\gamma$-quantiles of $\left(y_{\text {inf }}^{i}\right)_{i}$ and $\left(y_{\text {sup }}^{i}\right)_{i}$ respectively, as $\{y \mid \underline{F}(y) \geq \gamma\} \subseteq\{y \mid F(y) \geq \gamma\} \subseteq\{y \mid \bar{F}(y) \geq \gamma\}$.

\subsection{Rockfall simulation model: Rockyfor $3 D$}

The rock propagation simulations are conducted using the code Rockyfor3D. This well-known and widely used tool was developed in Irstea Grenoble conjointly with the Ecorisq research fondation (Dorren et al. 2006, Bourrier et al. 2009, Dorren 2015). Rockyfor3D allows simulating the $3 \mathrm{D}$ propagation of the rocks as a succession of free flights through the air and rebounds on the soil. The rock propagation is modelled through a rasterized Digital Terrain Model which can be seen as a matrix in which each cell value represents the mean terrain elevation of the square cell.

The set up of a rockfall simulation series requires defining a set of parameters related with the slope surface, the rocks and the release conditions (rock volume, departure location and initial falling height, in particular).

The rebound calculation is the keypoint for the assessment of rocks propagation. In the rockfall simulation code Rockyfor3D, it is conducted in two independent phases. First, the deviation of the block trajectory after the rebound is assessed and, second, the block reflected velocity is calculated. This modelling approach was shown to provide satisfying results at the slope scale (Dorren et al. 2006, Bourrier et al. 2009). One can note that the parameters related with the rebound calculation are modelled as independent random distributions. The rebound of the rock is modelled as a random process, i.e. for each rebound a set of parameters is sampled.

Once the simulation scenario defined, a series of rockfall simulations consists of releasing from each user-defined departure cell a given number of rocks one after the other. Output results from the simulations are used to build the complete statistical distribution of the rock trajectories (passing heights, kinetic energies ...) when entering a user-defined set of cells, corresponding to the fence location in this case.

\subsection{Probabilistic modelling of DEM uncertainty: ModTer}

The ModTer software (Crimier et al. 2016) aims to build a random field to model altimetric uncertainty,

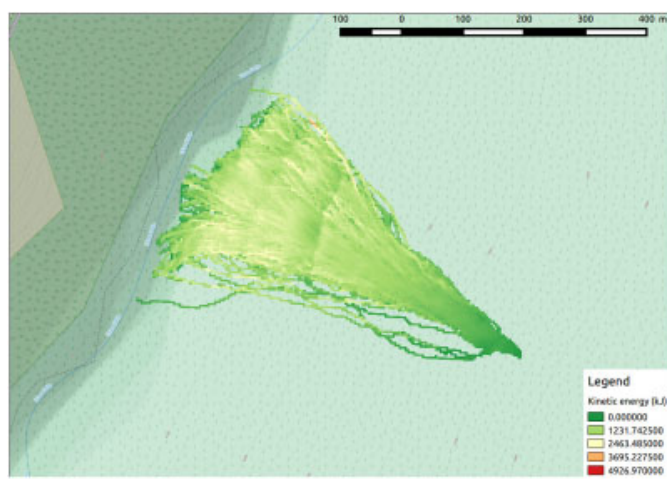

Figure 5. Kinetic energy realization (single RockyFor3D simulation)

from a given operational input Digital Elevation Model.

The field's parameters are set from statistical learning, using morphometric characteristics of the input DEM, and the errors between the input DEM and a set of available, reference altimetric data (Lidar, photogrammetry ...).

Once the model is build, the software is able to export a sample of output DEM, stemming from the random field.

\subsection{Description of the analysis}

Dupouy et al. (2015) aimed to apply and compare both Monte-Carlo and Hybrid approaches to propagate uncertainty and imprecision in the context of rockfall protective structure design. Nevertheless, this first study only focused on propagating uncertainty from thematic, scalar variables.

The aim of this study is to complete this previous work, by:

- modelling and propagating uncertainty on spatialized, input variables, namely the elevation model defining the local topography.

- dealing with a spatialized output variable of interest.

- evaluating the influence of elevation uncertainty on the resulting variable of interest, by leading a sensitivity analysis.

The study site corresponds to a full-scale rockfall experimental site located in an avalanche track in the forest owned by the local community Vaujany in Isère department, France (North $45^{\circ} 12^{\prime}$, East $6^{\circ} 3^{\prime}$ ).

A single departure cell is set: boulders start falling from this point.

The spatialized variable of interest is the kinetic energy 0.95 -quantile $E_{0.95}$. An example of this output raster, for a single RockyFor3D run on the study site, is shown in Figure 5.

In a cautious approach for hazard evaluation, the global uncertainty analysis' criteria of interest $c$ is the 0.975 -quantile of $E_{0.95}$.

Input uncertainties are set for both DEM, describing the topography, and the parameters related to the rock 


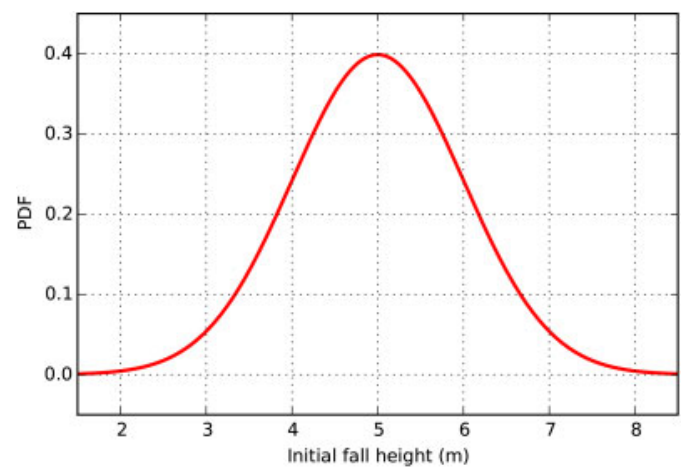

Figure 6. Fall height truncated Normal probability distribution (Monte Carlo/Hybrid propagation)

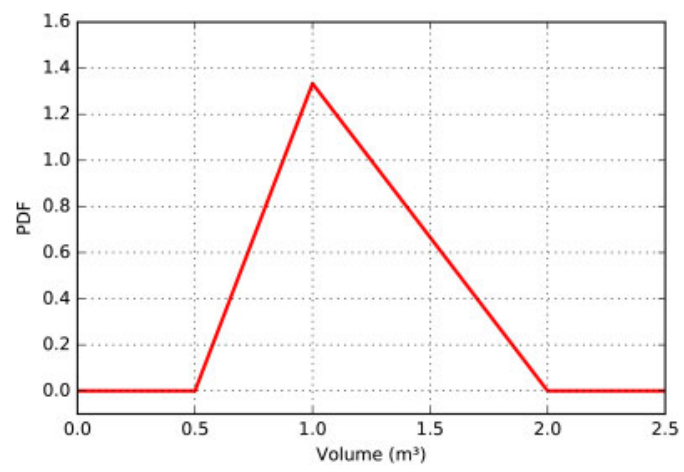

Figure 7. Volume Triangular probability distribution (Monte Carlo propagation).

release conditions, namely initial fall height and volume. The assumptions for these lasts thematic inputs are concordant with Dupouy et al. (2015):

- DEM's uncertainty is modelled using the ModTer tool. The same resulting stochastic field is used for both propagation method.

- $H$ is modelled using a single probability distribution for both cases. The values for $H$ are sampled from a truncated normal distribution with $\mu=5$ and $\sigma=1$, so that $H \in[0,10]$ (Figure 6).

- $V$ being the most important thematic parameter (Bourrier et al. 2009), the comparison between probabilistic and possibilistic approaches focuses on this parameter only. For the Hybrid case, the assumption of monotone relationship between $E_{0.95}$ and $V$ is reasonable.

We assume that expert knowledge is the only available source of information for quantifying $V$. The expert provides a bounded support interval $V \in[0.5,2]$, and a "central value": $V=1 \mathrm{~m}^{3}$, interpreted as a mode. Let us suppose then that the distribution of $V$ is uni-modal.

In a probabilistic paradigm, this information is usually traduced by a triangular probability distribution with support [0.5,2] and mode 1 (Figure 7).

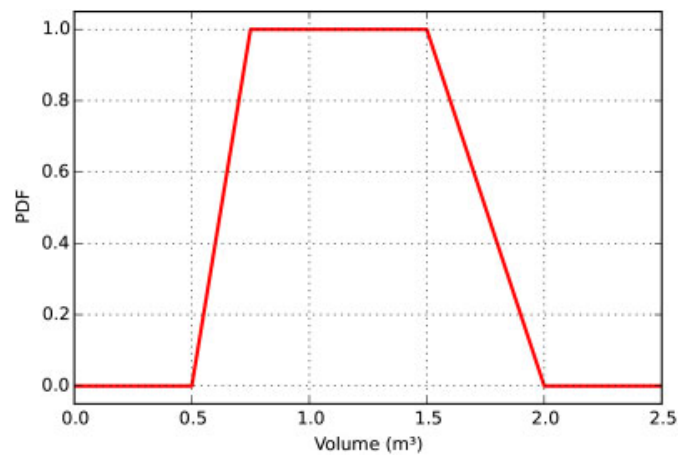

Figure 8. F:/Web/09-T-F-NL/Walls-ESREL-2450398/CH038/Volume Trapezoidal possibility distribution (Hybrid propagation).

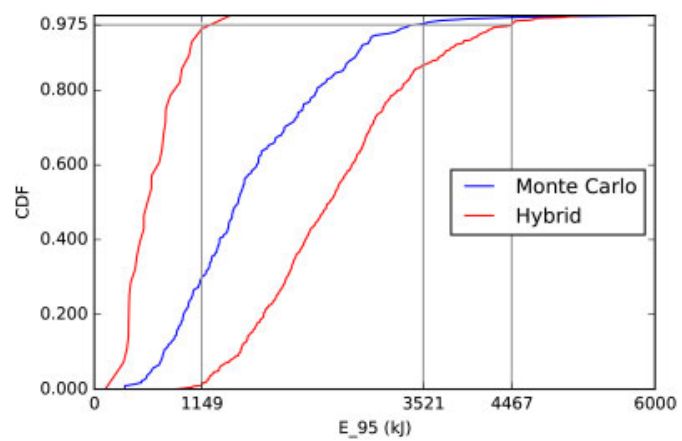

Figure 9. Comparison of Monte Carlo/Hybrid propagation in a specific raster cell: empirical distribution functions and 0.975 -quantiles of $E_{0.95}$.

In a possibilistic paradigm, as no symmetry hypothesis can be made here, according to Baudrit et al. (2003), a trapezium possibility distribution with support $[0.5,2]$ and mode $[0.75,1.5]$ (Figure 8 ) is tested.

\section{RESULTS}

\subsection{Uncertainty propagation}

The variable of interest is a spatialized output (see Figure 5), and each realization of $E_{0.95}$ is a raster file. The criterion of interest is estimated from a sample of 1000 rock propagation realizations.

That is to say, in each cell of the raster, simulations provide a scalar sample of local kinetic energy values. The 0.975-quantile is estimated in each cell, defining the global, rasterized estimator. The Figure 9 illustrates such cell-wise, scalar estimation.

\subsubsection{Monte Carlo propagation}

The quantile of interest estimated from Monte Carlo analysis is illustrated in Figure 10.

\subsubsection{Hybrid propagation}

The quantile of interest estimated from Hybrid analysis is bounded between the values represented in Figure 11 and those shown in Figure 12. 


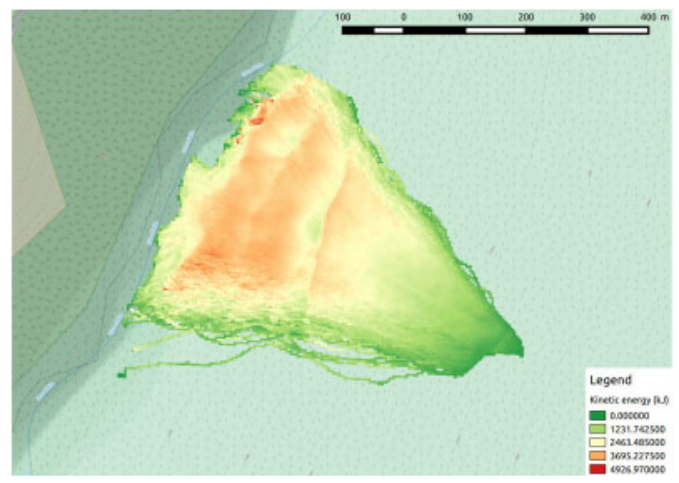

Figure 10. Monte Carlo propagation (stochastic DEM): Kinetic energy 0.975-quantile estimator.

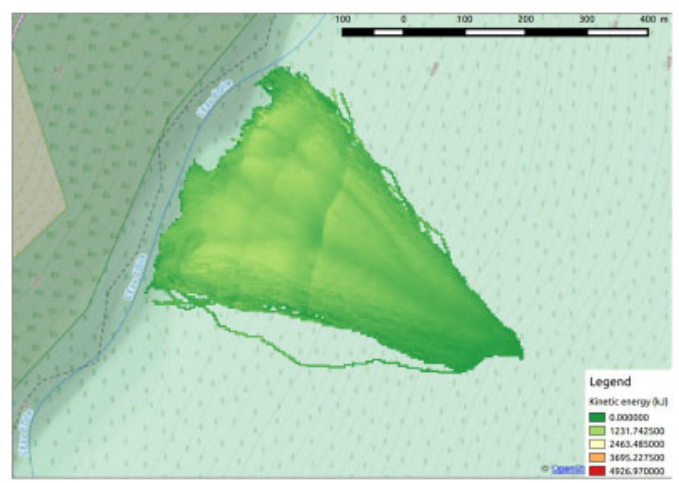

Figure 11. Hybrid propagation (stochastic DEM): Kinetic energy 0.975-quantile estimator's lower bound.

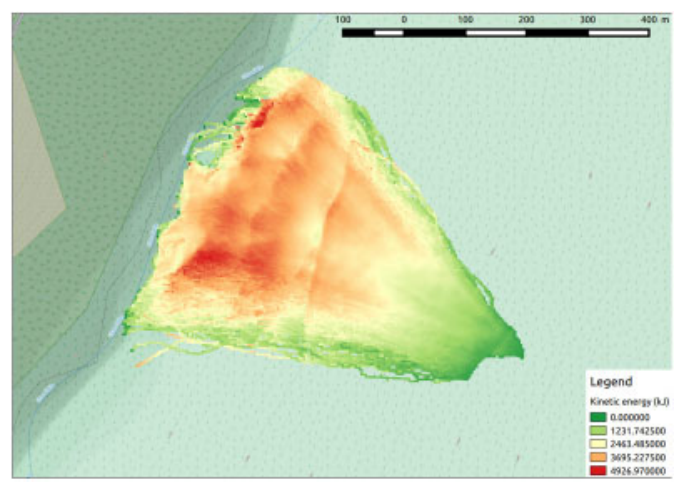

Figure 12. Hybrid propagation (stochastic DEM): Kinetic energy 0.975-quantile estimator's upper bound.

The (locally huge!) gap between lower and upper bounds reflects the imprecision of available information about input rock volume.

\subsection{Sensitivity of kinetic energy to elevation variability}

A Monte Carlo propagation has been done with a constant DEM. The quantification hypotheses for

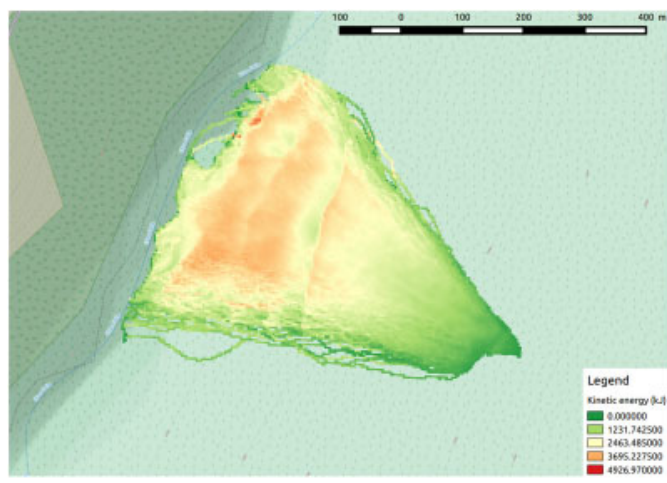

Figure 13. Monte Carlo propagation (constant DEM): Kinetic energy 0.975 -quantile estimator.

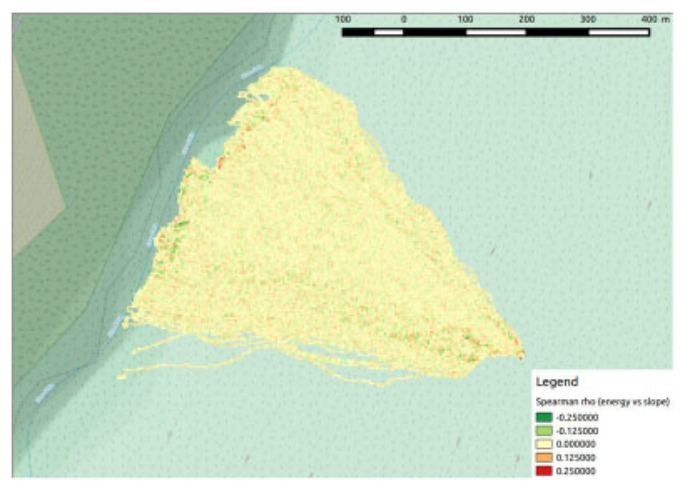

Figure 14. Spearman rho: Kinetic energy vs Slope.

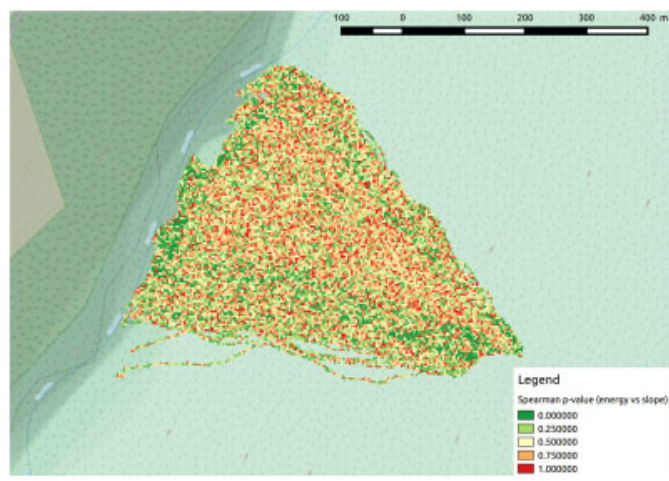

Figure 15. Spearman rho's P-value: Kinetic energy vs Slope.

thematic input variables (namely $H$ and $V$ ) are the same than in 3.1.1.

Figure 13 shows few differences compared to Figure 10: values for the quantile of interest are slightly lower, and the spread is less important.

The Spearman rho correlation indices between $E_{0.95}$ output and slope input samples, and the corresponding P-values, have been computed cell-wise. The Figure 14 (resp. Figure 15) illustrates the results of this sensitivity analysis. 


\subsection{Uncertainty propagation}

Dupouy et al. (2015) provided a first comparison between Monte Carlo and Hybrid propagation processes, to estimate a design value of a localized protective structure under deep uncertainty. It focused on a scalar variable of interest (kinetic energy at a protective fence location), and didn't take into account any DEM variability. Though it showed the interest of Hybrid methodology when dealing with imprecise information.

The conclusions are similar in this work, which calculates a spatialized estimation of the rockfall hazard, potentially providing expert support for estimating design values for a protective structure, whatever its position on the exposed area:

- The Hybrid approach provides large intervals bounding the high-level quantiles of interest of $E_{0.95}$. It is not mandatory to favour any value within these ranges, for no assumption is made about the true, unknown, probability distribution. The imprecision of these results reflects the quality of available information.

- The classical Monte Carlo approach provides a more convenient estimator, for it gives an single value as a criterion of interest (the estimator itself, or the upper bound of a confidence interval). Though, as the input quantification hypotheses rely on strong assumptions regarding the available information, its results shall be carefully trusted.

- In a concrete risk management, or protective structure design situation, the lower bound of the criterion provided by the Hybrid analysis defines a minimum design. The upper value provides the safer alternative, whatever the bet one may make on input uncertainties. Though, if any constraint makes it unaffordable, a Monte Carlo analysis with careful input quantification bet can provide a relevant design value. In such situation, both methods are complementary.

\subsection{Sensitivity of kinetic energy to elevation variability}

No global significant correlation can be assessed from the Spearman rho indicators, between the variable of interest $E_{0.95}$ and the slope variable deduced from the elevation raster sample.

A possible explanation is that boulders don't interact with local topography during free flight, but only during rebounds. It confirms the input rock volume to remain the main leading parameter (Bourrier et al. 2009) for rockfall kinetic energy.

Though, more relevant sensitivity indicators may be of interest, as Spearman rank correlation is best fit for evaluating monotone relationships.

\subsection{Future works}

This work has proposed an application of uncertainty and sensitivity analysis using probabilistic and possibilistic approaches to evaluate rockfall hazard and to assess the influence of altimetric uncertainty. Is it worth getting very precise spatial when uncertainty about others parameters is very high? We can imagine that the quality of spatial data will be of higher importance in case of very flat areas exposed to floods. Our approach and methodology will be extended to other natural phenomena such as floods in plain and mountains areas. This new framework and methodology will have to be improved to cope with heavy calculation time requirements. Those approaches are part of a global integrated framework dealing with information imperfection assessment and propagation in the decision process (Tacnet et al. 2014).

\section{ACKNOWLEDGMENTS}

Those developments have been partially funded under the framework of ModTer dual project funded by the DGA.

\section{REFERENCES}

Baudrit, C., D. Dubois, \& H. Fargier (2003, novembre). Représentation de la connaissance probabiliste incomplète. In Actes Rencontres Francophones sur la Logique Floue et ses Applications (LFA'2003), Tours, France, 26/11/2003-27/11/2003, pp. 65-72. Cepadues-Editions.

Baudrit, C., D. Guyonnet, \& D. Dubois (2006). Joint propagation and exploitation of probabilistic and possibilistic information in risk assessment. IEEE Transactions 14, 593-608.

Bourrier, F., L. Dorren, F. Nicot, F. Berger, \& F. Darve (2009). Toward objective rockfall trajectory simulation using a stochastic impact model. Geomorphology 110(3-4), 68-79.

Carladous, S., J.-M. Tacnet, J. Dezert, \& M. Batton-Hubert (2015). Belief function theory based decision support methods: Application to torrent protection work effectiveness and reliability assessment. In L. Podofillini, B. Sudret, B. Stojadinović, E. Zio, and W. Kröger (Eds.), Safety and Reliability of Complex Engineered Systems ESREL 2015 conference proceedings, 7-10 september, Zurich, London, UK, pp. 3643-3652. Taylor and Francis Group.

Crimier, N., K. Mekhnacha, J.-M. Tacnet, \& M. Memier (2016). Terrain uncertainties modelling software: Modter. In submitted to ESREL 2016 conference, Glasgow, Scotland.

de Cooman, G. \& D. Aeyels (1999). Supremum preserving upper probabilities. Information Sciences 118(1-4), 173-212.

Dempster, A. P. (1967, 04). Upper and lower probabilities induced by a multivalued mapping. Annals of Mathematical Statistics 38(2), 325-339.

Dorren, L. (2015). Rockyfor3d (v5.2) revealed - transparent description of the complete $3 \mathrm{~d}$ rockfall model. Technical report, ecorisQ (www.ecorisq.org).

Dorren, L., F. Berger, \& U. Putters (2006). Real-size experiments and 3-d simulation of rockfall on forested and non-forested slopes. Natural Hazards and Earth System Sciences 6(1), 145-153.

Dubois, D., H. NGuyen, \& H. Prade (2000). Fundamental of Fuzzy Sets, Chapter Possibility theory, probability 
and fuzzy sets : Misunderstandings, bridges and gaps, pp. 343-438. The Handbook of Fuzzy Sets Series. Boston, Massachussets, USA: Kluwer Academic Publishers.

Dubois, D. \& H. Prade (1992). When upper probabilities are possibility mesures. Fuzzy Sets and Systems 49, 65-74.

Dupouy, G., F. Bourrier, \& J.-M. Tacnet (2015). Uncertainty propagation for rockfall hazard modelling, comparison of monte carlo propagation method and hybrid approaches. In L. Podofillini, B. Sudret, B. Stojadinović, E. Zio, and W. Kröger (Eds.), Safety and Reliability of Complex Engineered Systems - ESREL 2015 conference proceedings, 7-10 september, Zurich, London, UK. Taylor and Francis Group.

Shafer, G. (1976). A mathematical theory of Evidence. Princeton University Press.
Smets, P. \& R. Kennes (1994). The transferable belief model. Artificial Intelligence 66(2), 191-234.

Tacnet, J.-M. (2009). Prise en compte de l'incertitude dans l'expertise des risques naturels en montagne par analyse multicritères et fusion d'information. Thèse de doctorat en sciences et génie de l'environnement, Ecole Nationale Supérieure des Mines, Saint-Etienne, France.

Tacnet, J.-M., J. Dezert, C. Curt, M. Batton-Hubert, \& E. Chojnacki (2014). How to manage natural risks in mountain areas in a context of imperfect information? new frameworks and paradigms for expert assessments and decision-making. Environment Systems and Decisions 34(2), 288-311.

Zadeh, L. A. (1978). Fuzzy sets as a basis for a theory of possibility. Fuzzy Sets and Systems 1, 3-28. 